Open Access

\title{
Development of Drug Resistance in Trichomonas vaginalis and its Overcoming with Natural Products
}

\author{
Stefanie Gehrig ${ }^{1}$ and Thomas Efferth ${ }^{2, *}$ \\ ${ }^{1}$ Institute of Pharmacy and Molecular Biotechnology, University of Heidelberg, Im Neuenheimer Feld 234, 69120 \\ Heidelberg \\ ${ }^{2}$ German Cancer Research Center, Pharmaceutical Biology (C015), Im Neuenheimer Feld 280, 69120 Heidelberg, \\ Germany
}

\begin{abstract}
Trichomoniasis is an infectious disease afflicting women worldwide. The protozoan parasite Trichomonas vaginalis is the causative agent of this sexually-transmitted disease, including also men in its infection cycle. The disease is usually not life-threatening, but has been associated with the development of cervical cancer and increased susceptibility to HIV. Approved drugs are 5-nitroimidazoles, with metronidazole being the drug of first choice. These drugs act via induction of oxidative stress and DNA-damage, leading to cell death in the parasite. Nevertheless, with the development of resistant $T$. vaginalis strains the treatment of the disease becomes exceedingly difficult. Mechanisms of drug resistance are characterized by reduced expression or even loss of proteins necessary for drug activation and a decreased reductive nature in the parasite. A promising strategy for research into new drugs and moreover, to overcome drug resistance, are compounds derived from natural sources. The present study provides a summary of all so far investigated small molecules with antitrichomonal activity; promisingly, some also show efficacy against resistant strains. Whereas the list of chemically characterized compounds derived from plants is rather short, literature provides immense applications of crude plant extracts tested against $T$. vaginalis. This demonstrates the absence of studies in this field aimed to identify and isolate single natural products exhibiting antitrichomonal features. Likewise, elucidating their mode of action on a molecular basis is of paramount importance.
\end{abstract}

Key Words: Anti-trichomonal activity, Chemotherapy, Drug resistance, Metronidazole, Natural products, Pharmacognosy, Trichomonas vaginalis, Trichomoniasis.

\section{INTRODUCTION}

Protozoal diseases are a major public health problem, predominately for people in tropical developing countries. The absence of proper medical facilities in these rural areas heightens their transmission [1]. Infections with drugresistant strains are one of the overriding problems in the fight against infectious diseases. Considering the limited drugs in current chemotherapy, which often act by the same mechanisms, no other drugs are available, if strains were to develop resistance. The search for new drugs is cost- and time-consuming, and drug development generally takes years. The pragmatic strategy is derivation from approved drugs to achieve improved pharmacological features. However, the mode of action remains the same and resistant strains do frequently not reveal sufficient susceptibility. Another problem is that standard chemotherapeutics often exhibit unconfirmed risks to patients. Due to their serious side effects, maximal dosages, needed for complete eradication of the protozoan parasite, and thereby, avoiding development of resistance, are deleterious [2]. This shows a strong need for research focused on new drugs to treat infectious diseases, to develop safe, effective drugs, and to

*Address correspondence to this author at the Cancer Research Center, Im Neuenheimer Feld 280, 69120 Heidelberg, Germany; Tel: 49-6221-423426; Fax: 49-6221-423433; E-mail: t.efferth@dkfz.de overcome drug resistance. Active substances can chemically be synthesized or, alternatively, can also be isolated from natural sources with high diversity of chemical structures. The most common of which is the use of plant-derived compounds in medical application, but fungi, bacteria, and animals also represent valuable sources for chemical substances with potency for treatment of diseases [3]. Of special interest are compounds with low cytotoxicity to host tissues and high antiprotozoal specificity. By serving as novel lead compounds, their structures can be optimized by semi-synthetic or fully synthetic derivatization [4]. In the fields of pharmacology and pharmacy, natural products have always played an important role in combating diseases. Famous examples are the glycosides digitoxin, isolated from Digitalis purpurea and digoxin, derived from $D$. lanata, which are applied against cardiac diseases. Other examples are demonstrated by the alkaloids vincristine and vinblastine, purified from the plant Vinca rosea (Catharanthus roseus) and paclitaxel, isolated from Taxus brevifolia, which are naturally derived anticancer drugs [1]. These successes exemplarily demonstrate that natural products are a potent source for the development of drugs against a variety of diseases, and in particular, to give impact on the treatment of trichomoniasis. Another strategy to combat infectious diseases such as trichomoniasis is vaccination. In the present review, vaccination is not covered. As an overview, key 
reviews that include insight on the development and progress of vaccination against $T$. vaginalis are recommended $[5,6]$.

\section{DISEASE AND CLINICAL MANIFESTATION}

Trichomonas vaginalis is a flagellated protozoan parasite of humans [7]. During sexual intercourse, it adheres to the epithelial cells of the genital tract and divides via binary fission to establish infection [6]. Trichomoniasis is the most common non-viral sexually transmitted disease (STD) [8], annually causing 174 million infections worldwide [9]. Due to the fact that the carrier stays asymptomatic during an incubation period of 4-28 days, transmission of the parasite often goes unnoticed [6, 10]. In women, infections can persist for long periods in the uro-genital tract along with mild symptoms like puritus and vaginal secretion [11]. In contrast, vaginitis and pelvic inflammatory diseases are characteristic symptoms for the acute inflammatory stage of the disease [12]. Moreover, cervical erosion [13], cervical cancer and infertility have been associated with trichomoniasis. Unborn children are also put at risk. Maternal inflammation often leads to premature rupture of the placental membranes causing pre-term delivery, low-birth-weight babies [7, 12, 14] and high infant mortality [15, 16]. Although the parasite can be transmitted to babies by breastfeeding, the children's oestrogen level which wanes in the third to sixth week of an infant's life prevents the development of a neonatal form of the disease [17]. In contrast to the adverse disease progression in women, spontaneous resolution of the infection in men is common [11]. The infection in men often remains dormant, implying their impact on disease transmission. The mild symptoms are characterized by discharge and itching. Only rarely the uro-genital tract is more seriously effected, which may result in infertility $[18,19]$. It is hypothesized that the parasite's pathogenesis is inhibited by the oxidative nature of the male genital tract [20]. Moreover, the prostatic fluid contains zinc which has been demonstrated to be cytotoxic to the parasite [21]. The female genital tract, on the contrary, provides a reducing environment which promotes development of the infection [20]. Although the low $\mathrm{pH}$ of the vagina impairs the parasite's cell detaching factors, $T$. vaginalis affords to erase Lactobacilli during infection. As a result, the $\mathrm{pH}$ increases and offers a better environment for invasion and growth of the parasite [22]. Menstrual blood also increases the $\mathrm{pH}$ and serves as iron supply to enhance the attachment of $T$. vaginalis [23]. Of particular relevance is the increased risk of human immunodeficiency virus (HIV) co-infection facilitated by local inflammation and microscopic breaches in mucosal barriers [24]. Furthermore, trichomoniasis causes degradation of a secretory leukocyte protease inhibitor, which normally protects cells from HIV infection [25].

\section{METABOLIC PECULIARITIES AS POTENTIAL DRUG TARGETS}

In order to approach the defense against the protozoan parasite, differences in biochemical mechanisms between parasite and host are promising drug targets without expected cytotoxicity towards the human host [10]. A particularity of Trichomonas is constituted by the lack of mitochondria, which renders energy metabolism anaerobic
[26]. Trichomonas compensates the less efficient metabolic processes by salvage pathways, demonstrated by a takeover of amino acids $[6,27]$, nucleotide precursors [28] and lipids [6]. Hydrogenosomes partially substitute mitochondria by representing a compartmentalized machinery for part of the energy metabolism [29]. The organelle's name is derived from its ability to produce hydrogen gas $\left(\mathrm{H}_{2}\right)$ [5]. In aerobic eukaryotes the oxidation of pyruvate is catalyzed in the mitochondria by pyruvate dehydrogenase, whereas in hydrognosomes $\mathrm{Fe}-S$-cluster proteins catalyze the production of ATP by oxidation of pyruvate to acid end products [10]. In the parasite, three proteins, namely pyruvate:ferredoxin oxidoreductase (PFOR), ferredoxin and Fe-hydrogenase, act in sequence to promote the metabolism of pyruvate. These proteins are highlighted in the following, because they also play an important role for the antitrichomonal action of current chemotherapeutics. The first enzyme in the cascade, PFOR, is also found in the cytosol of eukaryotes, but in $T$. vaginalis it is embedded in the hydrogenosomal membrane [30]. The second enzyme is ferredoxin, which shows a relationship to ferredoxins of the cytochrome P450-linked mixed-function oxidase systems of bacterial and vertebrate mitochondria [31]. The monomeric Fe-hydrogenase, presenting the third enzyme in the reaction, is also found in anaerobic bacteria, as well as in other aerobic/microaerophilic protozoan parasites, ciliates, fungi and chytrids. In the genome of $T$. vaginalis three Fe-hydrogenases have been identified with one being hypothetically localized in the hydrogenosome [32]. The cascade catalyzed by the three described enzymes is characterized by passing on of electrons. In the reaction, PFOR is reduced by oxidation of pyruvate, while coenzyme A is acetylated to form acetyl coenzyme A. Afterwards, PFOR reduces ferredoxin, and finally, the electrons are released to $\mathrm{Fe}$-hydrogenase, which produces hydrogen gas $\left(\mathrm{H}_{2}\right)$ by transferring electrons to protons [33]. Pyruvate is also formed by hydrogenosomal $\mathrm{NAD}^{+}$dependent malic enzyme [34]. NADH, generated in this reaction, is recycled by the reduction of ferredoxin catalyzed by NADH dehydrogenase. This enzyme was identified as functional homologue of the catalytic mode of the mitochondrial NADH dehydrogenase in complex I, the first step of the respiratory chain in mitochondria [35]. The NADH:ferredoxin oxidoreductase (NADH:FOR) activity of NADH hydrogenase presents an PFOR-independent pathway for drug activation, as well as the potential to maintain the redox balance [35]. An alternative mechanism for the pyruvate metabolism is constituted in the cytosol by lactate dehydrogenase, resulting in formation of lactate. Interestingly, lactate dehydrogenase is found in $T$. vaginalis, whereas it is missing Tritrichomonas foetus, the causative agent of trichomoniasis in cattle [29]. A further peculiarity of the parasite, and thereby presenting an additional drug target, is the biosynthesis of $\mathrm{Fe}-\mathrm{S}$-cluster proteins, with the necessity of cysteine as critical building block [36, 37]. Unlike the human host, Trichomonas can synthesize cysteine de novo by assimilation of sulphur from the environment followed by catalysis via cysteine synthase, a protein absent in mammals [27, 38]. However, Trichomonas offers no sulfur-containingamino acid metabolism, and additionally, it lacks the transsulfuration pathway to transform cysteine to methionine, or vice versa [39]. To compensate, the parasite possesses a 
unique enzyme for sulfur-containing amino acid degradation, methionine gamma-lyase (MGL) [27]. This protein has revealed a potency for drug development, since the compound L-trifluoromethionine (TFM), which is activated by MGL, was shown to be an efficient growth inhibitor in vitro against $T$. vaginalis [40] and E. histolytica [41], another human protozoan parasite sharing various biological and biochemical characteristics. Unfortunately, the drug target of the derived active component has not been elucidated yet. However, it was demonstrated that complete inhibition of MGL in vitro was not deleterious towards E. histolytica [41], reinforcing the assumption that MGL activity might also not be essential for $T$. vaginalis. Nevertheless, the efficiency of TFM was confirmed by a previous in vivo study with T. vaginalis infected mice [40]. Current research focuses on antitrichomonal derivatives of TFM along with development of new lead compounds [10].

\section{STANDARD CHEMOTHERAPY}

5-nitroimidazoles dominate the current chemotherapy of trichomoniasis. The drug of first choice is metronidazole [10], a synthetic 5-nitroimidazole developed in 1959 through derivation of the antibiotic azomycin isolated from Streptomyces eurocidicus [42-44]. The drug is administered orally or intravenously. A bioavailability of $93-100 \%$ is already achieved upon oral medication, because metronidazole does not bind to serum proteins [45]. The efficiency of chemotherapy is high, with a cure rate of $85-95 \%$ of treated patients and re-infections can be avoided through simultaneous treatment of sexual partners [17]. Chemotherapy using metronidazole commonly causes mild side effects, characterized by the body's defence mechanisms against toxic substances, like nausea, vomiting, diarrhoea, dizziness and headache. More serious side effects, like anorexia, hypersensitivity, leukopenia, palpitation, confusion, encephalopathy, and peripheral neuropathy are clinically rarely observed [46]. Nevertheless, metronidazole crosses the placental barrier and is, therefore, classified as a class B risk factor for pregnancy by the Food and Drug Administration (FDA), standing for an unconfirmed potential risk [5]. Even though, the risk of fetal exposure to metronidazole through treatment of the mother is considered to be less dangerous than the risk of the unborn child caused by maternal trichomoniasis [10]. To perform its mode of action metronidazole can enter the cell and its organelles via passive diffusion [10]. It is then activated by the reduced state of hydrogenosomal ferredoxin via reduction of the 5nitro group [47, 48]. This results in formation of a cytotoxic nitro radical anion which binds transiently to DNA to disrupt nucleotide strands, leading to cell death [49-52]. A similar mechanism of action is followed by tinidazole, another 5nitroimidazole compound, which is the drug of second choice in standard chemotherapy $[5,10]$. Due to an incomplete cross-resistance, tinidazole can be applied for treatment of metronidazole-resistant trichomoniasis [53, 54]. Further properties are its longer half-life and lower elimination rate compared with metronidazole, which might be the reason for its curative effect at lower dosages [55]. As a result, fewer and milder side effects occur using tinidazole for chemotherapy of trichomoniasis $[17,54,56]$.

\section{DEVELOPMENT OF RESISTANCE}

The development of drug resistance can be caused by different mechanisms. Altered metabolic pathways prevent the drug reaching its target. Alternative pathways facilitate the parasites' independence on inhibited targets or detoxification of the drug. In addition, intracellular accumulation of the drug can be inhibited by decreased drug import or increased drug export, thereby lowering the effective concentration below a threshold needed to harm the parasite [57]. Metronidazole resistance in T. vaginalis is described by two stages. Whereas these stages have been suggested to represent unrelated types of resistance formation, novel studies demonstrate a gradual transformation from the first stage to the second [58]. The aerobic resistance represents the earliest stage in resistance development, commonly observed in vivo by application of therapeutic levels of the drug [10]. It is characterized by reduction of oxygen scavenging processes. This results in an increased oxygen concentration inside the parasite impairing the low redox potential necessary for metronidazole activation [58-60]. In addition, activated metronidazole can be oxidized back to the inactive parent compound, a phenomenon called futile cycling. This reaction leads to the production of superoxide radical anions, causing limited cell damage compared to nitro radical anions produced via metronidazole activation [5, 61]. Furthermore, reduced expression levels of the hydrogenosomal enzymes PFOR, malic enzyme and NADH: FOR have been observed in the aerobic resistant stage, however, without affecting the level of activated metronidazole. Aerobic resistant strains were demonstrated to possess an increased sensitivity to oxidative stress. Thus exposition to high oxygen levels lead to cell death as a result of accumulation of toxic oxygen metabolites, which are normally prevented by effective oxygen scavenging processes $[59,60]$. Through further metabolic changes, the resistance develops to the anaerobic resistance stage [58], which was demonstrated in vitro by prolonged treatment and drug pressure [10]. This stage is oxygen-independent and characterized by advanced gradual loss of PFOR, the key enzyme in the drug activation pathway. However, alternative drug activation mechanisms via malic enzyme, NADH:FOR and ferredoxin still serve to activate the drug. To develop high resistance the hydrogenosome gets fully inactivated, realized by decrease and eventual loss of all proteins involved in drug activation, namely PFOR, malic enzyme, NADH:FOR, ferredoxin and Fe-hydrogenase [58, 62]. Down-regulation of PFOR and ferredoxin have also been demonstrated to result in resistance formation in Giardia intestinalis, an amitochondriate protozoan parasite of the intestinal tract [63-65]. Alteration of the ferredoxin level in aerobic resistant strains has been controversially presented in the literature. Whereas a decreased expression of the ferredoxin gene was found on the one hand [66], other studies demonstrate a constant level of ferredoxin in aerobic resistant $T$. vaginalis strains, as well as the enzymatic activity $[58,60,62]$. For protection from reactive oxygen species (ROS), Trichomonas possesses Fe-superoxide dismutase. This essential protein also presents a possible drug target, since it is absent in mammals [5]. Furthermore, resistant strains circumvent the necessity of inhibited 
proteins by switching to alternative pathways. The highly repetitive genome of Trichomonas, $160-280 \mathrm{Mb}$ in size, demonstrates that loss of a single gene might not be critical for survival of the parasite [10]. Exemplarily, the important enzyme PFOR can partially be replaced by two additional 2keto acid oxidoreducatases (KOR1 and KOR2) which are also involved in the energy metabolism [67]. KOR1 was shown to possess an increased activity in resistant strains and KOR2 is exclusively present upon development of resistance. Advantageously for resistant strains, metronidazole cannot be activated via the described mechanism while ferredoxin is still absent [10]. Besides the potential drug targets found up to date, no reasonable strategies are available for the increasing number of refractory cases caused by resistant $T$. vaginalis strains [5]. To fight persistent trichomoniasis, patients are treated with higher dosages of metronidazole over longer periods, resulting in deleterious side effects, intolerance and treatment failures [68, 69]. At least one percent of all infections with $T$. vaginalis cannot be cured, demonstrating the urgent need for the development of novel drugs to overcome drug resistance. The finding that tested resistant strains were sensitive to a non-nitroimidazole drug, the nitrofuran furazolidone [54], encourages the investigations for screening into new drugs representing different chemical structures. However, transmission of resistant $T$. vaginalis strains is not reported so far, and it is assumed that resistant strains are handicapped in a way which prevents transmission to another host. Therefore, development of resistance remains a problem for the carrier itself. This causes the assumption that there is no threat of epidemics caused by resistant $T$. vaginalis strains, and subsequently, generating no pressure for development of new drugs [65].

\section{SCREENING OF NATURAL PRODUCTS}

The potential of natural products in the treatment of diseases is evident in traditional medicines. Since treatments with medicinal plants were developed over thousands of years and are still practiced today, it might be promising to search for plants traditionally used to treat trichomoniasis and to isolate and characterize their bioactive chemical components [1]. Compared to other protozoan parasites, investigations of natural products with antitrichomonal activity are rarely found in the literature. Furthermore, most studies prescribe extracts, oils and tinctures of plants or marine organisms to exhibit an antitrichomonal activity. However, to permit meaningful investigations on a molecular level, it is important to focus on isolated compounds from plant raw extracts [70]. By restricting the present overview to compounds isolated from natural sources with activity against $T$. vaginalis, the subspecies of the genus Trichomonas being infectious to humans, the available studies are reduced to an unsatisfying minimum. In the following, the few compounds which exhibit activity against $T$. vaginalis published to date are organized according to their chemical structures. The antitrichomonal activities reported in the studies are presented, but a great disadvantage of almost all included investigations is the lack of concurrent proof of cytotoxicity to mammalian cells. Moreover, the reader should keep in mind that the data in the literature were generated in different experimental settings. Therefore, the values are not entirely comparable. Where possible, we indicated the activity of the reference drug, metronidazole, to allow the reader at least some comparability between different studies. The chemical structures of all compounds discussed in this review are shown in Fig. (1).

\section{Alkaloids}

The alkaloid berberine (1) and its salt, berberine sulphate, possessed in vitro activity against $T$. vaginalis. Berberine sulphate, isolated from Coptis teeta (Ranunculaceae), applied in a concentration of $1 \mathrm{mg} / \mathrm{ml}(2.3 \mathrm{mM})$ inhibited the growth of the parasite to $34.2 \%$ after $72 \mathrm{~h}$ of incubation. Subsequent analysis of the parasites revealed morphological changes, characterized by an increased number of vacuoles and/or their enlargement [71, 72]. In an additional study under the same experimental settings, berberine sulphate displayed a minimal lethal concentration (MLC) of 1.2

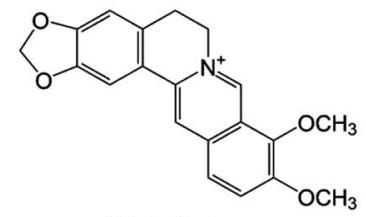

[1] berberine

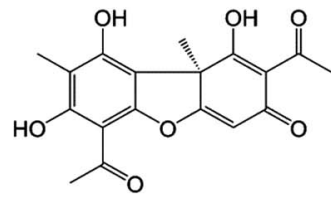

[2] S-(-)usnic acid

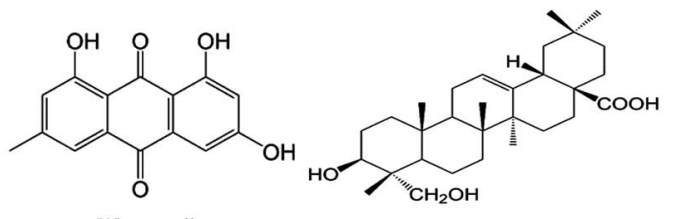

[3] emodin

[5] hederagin

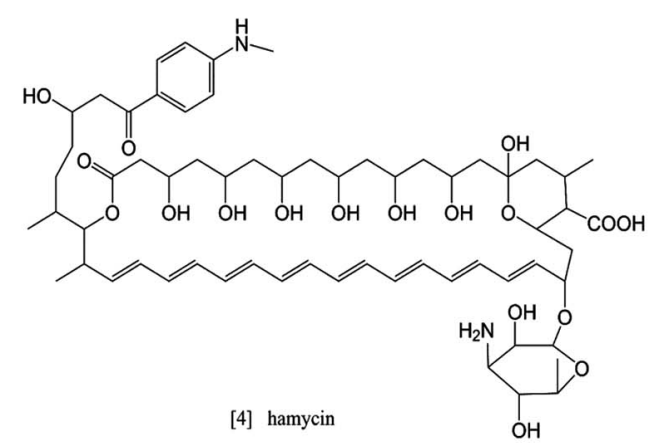

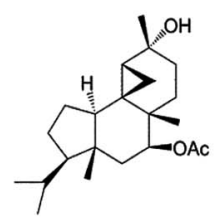

[6] azorellanol

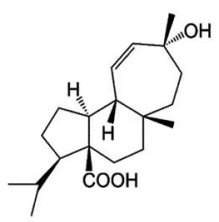

[7] mulinolic acid

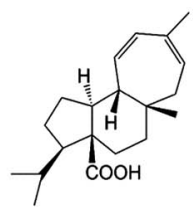

[8] mulin-11,13-dien-
20-oic acid

Fig. (1). Chemical structures of natural compounds with antitrichomonal activity. 
$\mathrm{mg} / \mathrm{ml}(2.7 \mathrm{mM})$. Furthermore, using incubation times of 24 and $48 \mathrm{~h}$ the effect of $1.2 \mathrm{mg} / \mathrm{ml}$ berberine sulphate was comparable to the reference drug metronidazole in a concentration of $25 \mu \mathrm{g} / \mathrm{ml}(146 \mu \mathrm{M})$. Afterwards, the effect of therapy cessation was investigated through reculturing of the parasites after drug treatment. Consequential, the same efficiency, indicated by no growth and loss of its motility, was observed after $72 \mathrm{~h}$ of incubation with either $1.2 \mathrm{mg} / \mathrm{ml}$ berberine sulphate or $25 \mu \mathrm{g} / \mathrm{ml}$ metronidazole. As a result, berberine sulphate possessed 18.5 -fold less antitrichomonal activity compared with metronidazole. Several modes of actions are suggested for the activity of berberine, and most likely it acts via intercalation into mitochondrial DNA, since it has been shown to function as an inhibitor of topoisomerase II while stabilizing topoisomerase II-DNA complexes [73].

\section{Phenolics}

Usnic acid, a dibenzofuran, is almost exclusively found in lichen and is especially abundant in genera such as Cladonia, Usnea, Lecanora, Ramalina and Evernia. S-(-)usnic acid (2) exhibited activity against $T$. vaginalis in vitro, with a lowest effective concentration of $0.4 \mathrm{mg} / \mathrm{ml}$ $(1.2 \mathrm{mM})$. The reference drug, metronidazole, displayed the same effects using $0.6 \mathrm{mg} / \mathrm{ml}(3.5 \mathrm{mM})$ [74, 75]. Accordingly, S-(-)usnic acid appears to be more potent for the treatment of trichomoniasis than the current chemotherapeutic agent metronidazole. However, the determined effective concentrations were rather high, namely in a millimolar range, whereas most of the literature demonstrates a micromolar concentration for the antitrichomonal activity of metronidazole.

The extract of Rheum valuate (Polygonaceae) exhibited lethal effects on Trichomonas in low concentrations (1:5000). In further investigations isolated compounds were tested independently during in vitro studies, revealing the anthraquinone emodin (1,3,8-trihydroxy-6-methylanthraquinone) (3) as active compound of the plant extract. Importantly, emodin affected $T$. vaginalis without possessing cytotoxicity towards mammalian cells. In an in vivo study with BALB/c mice, emodin was effective in decreasing subcutaneous abscesses caused by $T$. vaginalis infection when applied s.c. with $100 \mathrm{mg} / \mathrm{kg}$ body weight twice a day over a period of 7 days $(0.37 \mathrm{mM} / \mathrm{kg}$ body weight). Furthermore, emodin treatment delayed vaginal trichomoniasis for two days when applied s.c. using $100 \mathrm{mg} / \mathrm{kg}$ body weight twice a day $(0.37 \mathrm{mM} / \mathrm{kg}$ body weight). Also oral administration of emodin exhibited curative effects, but higher dosages had to be applied. The volume of abscesses was reduced when emodin was applied p.o. with $500 \mathrm{mg} / \mathrm{kg}$ body weight twice a day $(1.85 \mathrm{mM} / \mathrm{kg}$ body weight $)$, which was comparable with the application of metronidazole s.c. of $50 \mathrm{mg} / \mathrm{kg}$ body weight twice a day $(0.29 \mathrm{mM} / \mathrm{kg}$ body weight). The mode of action of emodin has not been studied extensively, but it was demonstrated that its antitrichomonal activity was reversed by catalase, a scavenger of hydrogen peroxide. Therefore, it is suggested that the antitrichomonal activity of emodin is mediated via production of hydrogen peroxide as toxic oxygen metabolite [76].

\section{Polyenes}

Hamycin (4), a heptaene, was isolated from Streptomyces pimprina (Streptomycetaceae). This compound is structurally related to amphotericin B and is known for its antifungal properties. Interestingly, hamycin is used in India for topical treatment of trichomoniasis. In field studies, topical application of hamycin revealed a cure rate of $88 \%$ in two infected women. Nevertheless, hamycin is prohibited to parental use due to its systemic toxicity. In addition, an animal model also indicated tissue toxicity observed upon topical use of hamycin. In an in vitro study the antitrichomonal activity of hamycin was carried out with a minimal inhibition concentration (MIC) of $1 \mu \mathrm{g} / \mathrm{ml}(0.87 \mu \mathrm{M})$ [77]. In further investigations, hamycin was proved in different formulations for activity against metronidazole-resistant and metronidazolesensitive T. vaginalis strains. The MIC values for metronidazole were $4-16 \mu \mathrm{g} / \mathrm{ml}(23.4-92.4 \mu \mathrm{M})$ for susceptible strains and increased to $200-1500 \mu \mathrm{g} / \mathrm{ml}(1168-8763 \mu \mathrm{M})$ concerning resistant strains. The $\mathrm{IC}_{50}$ value for liposomal formulation of hamycin was $1.2-2.4 \mu \mathrm{g} / \mathrm{ml}(1.04-2.08 \mu \mathrm{M})$, whereas hamycin-glycerine possessed a higher antitrichomonal activity with an $\mathrm{IC}_{50}$ value of $0.5-1.0 \mu \mathrm{g} / \mathrm{ml}(0.43-0.86 \mu \mathrm{M})$, but this value is critical, given that the latter formulation was incompletely solubilized. Use of hamycin-dimethylsulfoxid and hamycin-deoxycholate was also problematic due to instability of the formulations. Hamycin-dimethylsufoxid gels possessed the highest antitrichomonal activity with an $\mathrm{IC}_{50}$ value of $0.3-1.3 \mu \mathrm{g} / \mathrm{ml}(0.26-1.12 \mu \mathrm{M})$, but the formulation was only stable for less than $24 \mathrm{~h}$. Similar results have been observed for hamycin-deoxycholate gels, which freshly prepared exhibited an $\mathrm{IC}_{50}$ value of $0.5-1.0 \mu \mathrm{g} / \mathrm{ml}(0.43-$ $0.86 \mu \mathrm{M})$, whereas after storage of two months a decreased activity was prescribed. A further compound, amphotericin $\mathrm{B}$, exerted an antitrichomonal activity with an $\mathrm{IC}_{50}$ value of $12.5 \mu \mathrm{g} / \mathrm{ml}(13.5 \mu \mathrm{M})$. In addition, hamycin and amphotericin B were effective for treating both, metronidazolesensitive and -resistant, T. vaginalis strains. On the contrary, hamycin showed no specificity towards the parasite. Concentration of the drug applied for deleterious effects on $T$. vaginalis were also cytotoxic to the tested mammalian cell lines, BHK-21 and HeLa. This prohibits the medial use of this compound. The mode of action of hamycin is similar to that of other aromatic polyene antibiotics like amphotericin B. The mechanism involves binding to ergosterols present in the plasma membrane of eukaryotic cells, along with the formation of pores, leading to depletion of cytoplasm and potenial cell death [78].

\section{Terpenoids}

Hederagin (3,23-dihydroxy-12-oleanen-28-oic acid) (5), a pentacyclic triterpenoid, was isolated from Cussonia holstii (Araliaceae), a traditional medical plant used in Kenya. In vitro, the $\mathrm{IC}_{50}$ value of hederagin was $2.8 \mu \mathrm{M}$. However, metronidazol, was 10 times more efficient displaying an $\mathrm{IC}_{50}$ value of $0.28 \mu \mathrm{M}$ [79].

Diterpenoids with antitrichomonal activity were isolated from Azorella yareta (Apiaceae), a plant native in northeastern Chile and northwestern Argentina. The compounds azorellanol (6), mulinolic acid (7) and mulin-11,13-dien-20oic acid $(\mathbf{8})$ revealed $\mathrm{LD}_{50}$ values in the range of $40 \mu \mathrm{M}$. The 
reference drug, metronidazole, was six times more active, with an $\mathrm{LD}_{50}$ value of $6.6 \mu \mathrm{M}$ [80].

\section{CONCLUSION}

After prolonged exposure to drugs, parasites frequently develop resistance towards chemotherapeutics. Natural products present a promising source for the development of new drugs. Nevertheless, there is a lack of isolated compounds evaluated for their activity against trichomoniasis. The presented terpenoids $(\mathbf{5}, \mathbf{6}, \mathbf{7}, \mathbf{8})$ exhibited a satisfactory activity against the parasite. However, this study lacked determination of possible cytotoxicity of the compounds to mammalian cell lines, data of great importance to evaluate their potential as alternative antitrichomonal drugs. In general, evaluation of natural compounds in animal models, identification of their mode of action, as well as determination of resistance formation after extensive application is vastly missing in the literature. Up to date resistance formation in $T$. vaginalis towards natural products is not reported. The finding that $T$. vaginalis can rapidly develope resistance to zinc, an antitrichomonal component present in the prostatic fluid [21], demonstrates the likeliness that evolution has enabled parasites to develop resistance to substances they might have been exposed to. However, no presence of natural products is expected in the uro-genital tract. Given that a variety of plant extracts have demonstrated an antitrichomonal activity, research needs to identify their active components. The understanding of the molecular mode of action is of great importance to foster the development of potential new drugs. For example, use of microarray studies to show regulation of genes which are affected by drug treatment and to elucidate pathways in which the drug act. When a target molecule is known, site-directed mutagenesis or QSAR (quantitative structure-activity relationship)analysis are facilitating methods to reveal structure-activity relationships by investigation of the molecular properties which account for the biological action of a drug. However, only a small number of drug candidates have been investigated so far. This is the reason why drug development with compounds from natural sources has still had little impact on the treatment of trichomoniasis. To our opinion, there is a clear need for natural product research to improve the treatment of trichomonal infections.

\section{REFERENCES}

[1] Tagboto, S.; Townson, S. Antiparasitic properties of medicinal plants and other naturally occurring products. Adv. Parasitol., 2001, 50, 199-295.

[2] Kayser, O; Kiderlen, A.F. Delivery strategies for antiparasitics. Expert Opin. Investig. Drugs, 2003, 12(2), 197-207.

[3] Kayser, O.; Kiderlen, A.F.; Croft, S.L. Natural products as antiparasitic drugs. Parasitol. Res., 2003, 90(Suppl 2), S55-62.

[4] Holzgrabe, U.; Bechthold, A. Paradigmenwechsel in der Entwicklung antiinfektiver Chemotherapeutika. Chemother. J., 1999, 8(2), 68-78.

[5] Cudmore, S.L.; Delgaty, K.L.; Hayward-McClelland, S.F.; Petrin, D.P.; Garber, G.E. Treatment of infections caused by metronidazole-resistant Trichomonas vaginalis. Clin. Microbiol. Rev., 2004, 17(4), 783-793, Table of contents.

[6] Petrin, D.; Delgaty, K.; Bhatt, R.; Garber, G. Clinical and microbiological aspects of. Trichomonas vaginalis. Clin. Microbiol. Rev., 1998, 11(2), 300-317.

[7] Rein, M.F.; Chapel, T.A. In Trichomonads parasitic in humans; Honigberg, Ed.; Springer-Verlag: New York, 1990; pp. 225-234.
[8] Gerbase, A.C.; Rowley, J.T.; Heymann, D.H.; Berkley, S.F.; Piot, P. Global prevalence and incidence estimates of selected curable STDs. Sex. Transm. Infect., 1998, 74(Suppl 1), S12-6.

[9] World Health Organisation. Overviews and estimates: Global prevalence and incidence of selected curable sexually transmitted infections. http://www.who.int/hiv/pub/sti/en/who_hiv_aids_2001. 02.pdf (accessed May 2009)

[10] Ali, V.; Nozaki, T. Current therapeutics, their problems, and sulfurcontaining-amino-acid metabolism as a novel target against infections by "amitochondriate" protozoan parasites. Clin. Microbiol. Rev., 2007, 20(1), 164-187.

[11] Heine, P.; McGregor, J.A. Trichomonas vaginalis: a reemerging pathogen. Clin. Obstet. Gynecol., 1993, 36(1), 137-144.

[12] Rein, M.F.; Chapel, T.A. Trichomoniasis, candidiasis, and the minor venereal diseases. Clin. Obstet. Gynecol., 1975, 18(1), 7388.

[13] McLellan, R.; Spence, M.R.; Brockman, M.; Raffel, L.; Smith, J.L. The clinical diagnosis of trichomoniasis. Obstet. Gynecol., 1982, 60(1), 30-34.

[14] Wolner-Hanssen, P.; Krieger, J.N.; Stevens, C.E.; Kiviat, N.B.; Koutsky, L.; Critchlow, C.; DeRouen, T.; Hillier, S.; Holmes, K.K. Clinical manifestations of vaginal trichomoniasis. JAMA, 1989, 261(4), 571-576.

[15] Cotch, M.F.; Pastorek, J.G., 2nd; Nugent, R.P.; Hillier, S.L.; Gibbs, R.S.; Martin, D.H.; Eschenbach, D.A.; Edelman, R.; Carey, J.C.; Regan, J.A.; Krohn, M.A.; Klebanoff, M.A.; Rao, A.V.; Rhoads, G.G. Trichomonas vaginalis associated with low birth weight and preterm delivery. The Vaginal Infections and Prematurity Study Group. Sex. Transm. Dis., 1997, 24(6), 353-360.

[16] Saurina, G.R.; McCormack, W.M. Trichomoniasis in pregnancy. Sex. Transm. Dis., 1997, 24(6), 361-362.

[17] Lossick, J.G. Treatment of sexually transmitted vaginosis/vaginitis. Rev. Infect. Dis., 1990, 12(Suppl 6), S665-S681.

[18] Krieger, J.N. Prostatitis syndromes: pathophysiology, differential diagnosis, and treatment. Sex. Transm. Dis., 1984, 11(2), 100-112.

[19] Mardh, P.A.; Colleen, S. Search for uro-genital tract infections in patients with symptoms of prostatitis. Studies on aerobic and strictly anaerobic bacteria, mycoplasmas, fungi, trichomonads and viruses. Scand. J. Urol. Nephrol., 1975, 9(1), 8-16.

[20] Alderete, J.F.; Provenzano, D. The vagina has reducing environment sufficient for activation of Trichomonas vaginalis cysteine proteinases. Genitourin. Med., 1997, 73(4), 291-296.

[21] Krieger, J.N.; Rein, M.F. Zinc sensitivity of Trichomonas vaginalis: in vitro studies and clinical implications. J. Infect. Dis., 1982, 146(3), 341-345.

[22] Goode, M.A.; Grauer, K.; Gums, J.G. Infectious vaginitis. Selecting therapy and preventing recurrence. Postgrad. Med., 1994, 96(6), 85-8, 91-98.

[23] Lehker, M.W.; Arroyo, R.; Alderete, J.F. The regulation by iron of the synthesis of adhesins and cytoadherence levels in the protozoan Trichomonas vaginalis. J. Exp. Med., 1991, 174(2), 311-318.

[24] Schwebke, J.R.; Burgess, D. Trichomoniasis. Clin. Microbiol. Rev., 2004, 17(4), 794-803. 803, Table of content.

[25] Draper, D.; Donohoe, W.; Mortimer, L.; Heine, R.P. Cysteine proteases of Trichomonas vaginalis degrade secretory leukocyte protease inhibitor. J. Infect. Dis., 1998, 178(3), 815-819.

[26] Muller, M. In Molecular medical parasitology. Marr, N.W., Eds.; Academic Press: London, 2003; pp. 125-139.

[27] Nozaki, T.; Ali, V.; Tokoro, M. Sulfur-containing amino acid metabolism in parasitic protozoa. Adv. Parasitol., 2005, 60, 1-99.

[28] Wang, C.C.; Verham, R.; Tzeng, S.F.; Aldritt, S.; Cheng, H.W. Pyrimidine metabolism in Tritrichomonas foetus. Proc. Natl. Acad. Sci. USA, 1983, 80(9), 2564-2568.

[29] Kulda, J. Trichomonads, hydrogenosomes and drug resistance. Int. J. Parasitol., 1999, 29(2), 199-212.

[30] Williams, K.; Lowe, P.N.; Leadlay, P.F. Purification and characterization of pyruvate: ferredoxin oxidoreductase from the anaerobic protozoon Trichomonas vaginalis. Biochem. J., 1987, 246(2), 529-536.

[31] Johnson, P.J.; d'Oliveira, C.E.; Gorrell, T.E.; Muller, M. Molecular analysis of the hydrogenosomal ferredoxin of the anaerobic protist Trichomonas vaginalis. Proc. Natl. Acad. Sci. USA, 1990, 87(16), 6097-101. 
[32] Embley, T.M.; van der Giezen, M.; Horner, D.S.; Dyal, P.L.; Bell, S.; Foster, P.G. Hydrogenosomes, mitochondria and early eukaryotic evolution. IUBMB Life, 2003, 55(7), 387-395.

[33] Dyall, S.D.; Yan, W.; Delgadillo-Correa, M.G.; Lunceford, A.; Loo, J.A.; Clarke, C.F.; Johnson, P.J. Non-mitochondrial complex I proteins in a hydrogenosomal oxidoreductase complex. Nature, 2004, 431(7012), 1103-1107.

[34] Hrdý, I.; Cammack, R.; Stopka, P.; Kulda, J.; Tachezy, J. Alternative pathway of metronidazole activation in Trichomonas vaginalis hydrogenosomes. Antimicrob. Agents Chemother., 2005, 49(12), 5033-5036.

[35] Hrdý, I.; Hirt, R.P.; Dolezal, P.; Bardonova, L.; Foster, P.G.; Tachezy, J.; Embley, T.M. Trichomonas hydrogenosomes contain the NADH dehydrogenase module of mitochondrial complex I. Nature, 2004, 432(7017), 618-622.

[36] Beinert, H.; Holm, R.H.; Munck, E. Iron-sulfur clusters: nature's modular, multipurpose structures. Science, 1997, 277(5326), 653659.

[37] Johnson, D.C.; Dean, D.R.; Smith, A.D.; Johnson, M.K. Structure, function, and formation of biological iron-sulfur clusters. Аппи. Rev. Biochem., 2005, 74, 247-281.

[38] Westrop, G.D.; Goodall, G.; Mottram, J.C.; Coombs, G.H. Cysteine biosynthesis in Trichomonas vaginalis involves cysteine synthase utilizing O-phosphoserine. J. Biol. Chem., 2006, 281(35), 25062-25075.

[39] McKie, A.E.; Edlind, T.; Walker, J.; Mottram, J.C.; Coombs, G.H. The primitive protozoon Trichomonas vaginalis contains two methionine gamma-lyase genes that encode members of the gamma-family of pyridoxal 5 '-phosphate-dependent enzymes. $J$. Biol. Chem., 1998, 273(10), 5549-5556.

[40] Coombs, G.H.; Mottram, J.C. Trifluoromethionine, a prodrug designed against methionine gamma-lyase-containing pathogens, has efficacy in vitro and in vivo against Trichomonas vaginalis. Antimicrob. Agents Chemother., 2001, 45(6), 1743-1745.

[41] Tokoro, M.; Asai, T.; Kobayashi, S.; Takeuchi, T.; Nozaki, T. Identification and characterization of two isoenzymes of methionine gamma-lyase from Entamoeba histolytica: a key enzyme of sulfur-amino acid degradation in an anaerobic parasitic protist that lacks forward and reverse trans-sulfuration pathways. $J$. Biol. Chem., 2003, 278(43), 42717-42727.

[42] Cosar, C.; Julou, L. [The activity of 1-(2-hydroxyethyl)-2-methyl5-nitroimidazole (R. P. 8823) against experimental Trichomonas vaginalis infections.]. Ann. Inst. Pasteur (Paris), 1959, 96(2), 238241.

[43] Osato, T.; Ueda, M.; Fukuyama, S.; Yagishita, K.; Okami, Y.; Umezawa, H. Production of tertiomycin (a new antibiotic substance), azomycin and eurocidin by S. eurocidicus. J. Antibiot. (Tokyo), 1955, 8(4), 105-109.

[44] Nakamura, S. Structure of azomycin, a new antibiotic. Pharm. Bull., 1955, 3(5), 379-383.

[45] Houghton, G.W.; Smith, J.; Thorne, P.S.; Templeton, R. The pharmacokinetics of oral and intravenous metronidazole in man. $J$. Antimicrob. Chemother., 1979, 5(5), 621-623.

[46] Lossick, J.G. In Trichomonads Parasitic in Humans. Honiberg, B.M., Ed.; Springer-Verlag: New York, 1990; pp. 324-341.

[47] Lindmark, D.G. Energy metabolism of the anaerobic protozoon Giardia lamblia. Mol. Biochem. Parasitol., 1980, 1(1), 1-12.

[48] Muller, M. Reductive activation of nitroimidazoles in anaerobic microorganisms. Biochem. Pharmacol., 1986, 35(1), 37-41.

[49] Declerck, P.J.; De Ranter, C.J. In vitro reductive activation of nitroimidazoles. Biochem. Pharmacol., 1986, 35(1), 59-61.

[50] Edwards, D.I. Nitroimidazole drugs--action and resistance mechanisms. I. Mechanisms of action. J. Antimicrob. Chemother., 1993, 31(1), 9-20.

[51] Edwards, D.I. Nitroimidazole drugs--action and resistance mechanisms. II. Mechanisms of resistance. J. Antimicrob. Chemother., 1993, 31(2), 201-210.

[52] Lloyd, D.; Kristensen, B. Metronidazole inhibition of hydrogen production in vivo in drug-sensitive and resistant strains of Trichomonas vaginalis. J. Gen. Microbiol., 1985, 131(4), 849-853.

[53] Lossick, J.G.; Kent, H.L. Trichomoniasis: trends in diagnosis and management. Am. J. Obstet. Gynecol., 1991, 165(4 Pt 2), 12171222 .
[54] Narcisi, E.M.; Secor, W.E. In vitro effect of tinidazole and furazolidone on metronidazole-resistant Trichomonas vaginalis. Antimicrob. Agents. Chemother., 1996, 40(5), 1121-1125.

[55] Crowell, A.L.; Sanders-Lewis, K.A.; Secor, W.E. In vitro metronidazole and tinidazole activities against metronidazole-resistant strains of Trichomonas vaginalis. Antimicrob. Agents Chemother., 2003, 47(4), 1407-1409.

[56] Malla, N.I.; Gupta, I.; Sokhey, C.; Sehgal, R.; Ganguly, N.K.; Mahajan, R.C. In vitro evaluation of metronidazole and tinidazole on strains of Trichomonas vaginalis. Indian J. Med. Microbiol., 1988, 6, 297-301.

[57] Weber, J.T.; Courvalin, P. An emptying quiver: antimicrobial drugs and resistance. Emerg. Infect. Dis., 2005, 11(6), 791-793.

[58] Rasoloson, D.; Vanacova, S.; Tomkova, E.; Razga, J.; Hrdy, I.; Tachezy, J.; Kulda, J. Mechanisms of in vitro development of resistance to metronidazole in Trichomonas vaginalis. Microbiology, 2002, 148(Pt 8), 2467-2477.

[59] Ellis, J.E.; Yarlett, N.; Cole, D.; Humphreys, M.J.; Lloyd, D. Antioxidant defences in the microaerophilic protozoan Trichomonas vaginalis: comparison of metronidazole-resistant and sensitive strains. Microbiology, 1994, 140, 2489-2494.

[60] Rasoloson, D.; Tomkova, E.; Cammack, R.; Kulda, J.; Tachezy, J. Metronidazole-resistant strains of Trichomonas vaginalis display increased susceptibility to oxygen. Parasitology, 2001, 123(Pt 1), 45-56.

[61] Perez-Reyes, E.; Kalyanaraman, B.; Mason, R.P. The reductive metabolism of metronidazole and ronidazole by aerobic liver microsomes. Mol. Pharmacol., 1980, 17(2), 239-244.

[62] Kulda, J.; Hrdý, I. In Hydrogenosomes and Mitosomes: Mitochondria of Aerobic Eukaryotes. Tachezy, Ed.; Springer-Verlag: Berlin Heidelberg, 2007; pp. 179-196.

[63] Liu, S.M.; Brown, D.M.; O'Donoghue, P.; Upcroft, P.; Upcroft, J.A. Ferredoxin involvement in metronidazole resistance of Giardia duodenalis. Mol. Biochem. Parasitol., 2000, 108(1), 137140.

[64] Townson, S.M.; Upcroft, J.A.; Upcroft, P. Characterisation and purification of pyruvate:ferredoxin oxidoreductase from Giardia duodenalis. Mol. Biochem. Parasitol., 1996, 79(2), 183-193.

[65] Upcroft, P.; Upcroft, J.A. Drug targets and mechanisms of resistance in the anaerobic protozoa. Clin. Microbiol. Rev., 2001, 14(1), 150-164.

[66] Quon, D.V.; d'Oliveira, C.E.; Johnson, P.J. Reduced transcription of the ferredoxin gene in metronidazole-resistant Trichomonas vaginalis. Proc. Natl. Acad. Sci. USA, 1992, 89(10), 4402-4406.

[67] Brown, D.M.; Upcroft, J.A.; Dodd, H.N.; Chen, N.; Upcroft, P. Alternative 2-keto acid oxidoreductase activities in Trichomonas vaginalis. Mol. Biochem. Parasitol., 1999, 98(2), 203-214.

[68] Lewis, D.A.; Habgood, L.; White, R.; Barker, K.F.; Murphy, S.M. Managing vaginal trichomoniasis resistant to high-dose metronidazole therapy. Int. J. STD AIDS, 1997, 8(12), 780-784.

[69] Ti, T.Y.; Lee, H.S.; Khoo, Y.M. Disposition of intravenous metronidazole in Asian surgical patients. Antimicrob. Agents Chemother., 1996, 40(10), 2248-2251.

[70] Tasdemir, D.; Kaiser, M.; Brun, R.; Yardley, V.; Schmidt, T.J.; Tosun, F.; Ruedi, P. Antitrypanosomal and antileishmanial activities of flavonoids and their analogues: in vitro, in vivo, structureactivity relationship, and quantitative structure-activity relationship studies. Antimicrob. Agents Chemother., 2006, 50(4), 1352-1364.

[71] Kaneda, Y.; Tanaka, T.; Saw, T. Effects of berberine, a plant alkaloid, on the growth of anaerobic protozoa in axenic culture. Tokai J. Exp. Clin. Med., 1990, 15(6), 417-423.

[72] Kaneda, Y.; Torii, M.; Tanaka, T.; Aikawa, M. In vitro effects of berberine sulphate on the growth and structure of Entamoeba histolytica, Giardia lamblia and Trichomonas vaginalis. Ann. Trop. Med. Parasitol., 1991, 85(4), 417-425.

[73] Soffar, S.A.; Metwali, D.M.; Abdel-Aziz, S.S.; el-Wakil, H.S.; Saad, G.A. Evaluation of the effect of a plant alkaloid (berberine derived from Berberis aristata) on Trichomonas vaginalis in vitro. J. Egypt Soc. Parasitol., 2001, 31(3), 893-904.

[74] Wu, J.; Zhang, M.; Ding, D.; Tan, T.; Yan, B. [Effect of Cladonia alpestris on Trichomonas vaginalis in vitro]. Zhongguo Ji Sheng Chong Xиe Yu Ji Sheng Chong Bing Za Zhi, 1995, 13(2), 126129. 
[75] Lauterwein, M.; Oethinger, M.; Belsner, K.; Peters, T.; Marre, R. In vitro activities of the lichen secondary metabolites vulpinic acid, (+)-usnic acid, and (-)-usnic acid against aerobic and anaerobic microorganisms. Antimicrob. Agents Chemother., 1995, 39(11), 2541-2543.

[76] Wang, H.H. Antitrichomonal action of emodin in mice. $J$. Ethnopharmacol., 1993, 40(2), 111-116.

[77] Bhagwat, P.D.; Gokhale, B.B.; Sane, H.S.; Thirumalachar, M.J. Assessment of antitrichomonal activity of hamycin. Indian J. Med. Res., 1964, 52, 36-37.
[78] Lushbaugh, W.B.; Cleary, J.D.; Finley, R.W. Cytotoxicity of hamycin for Trichomonas vaginalis, HeLa and BHK-21. J. Antimicrob. Chemother., 1995, 36(5), 795-802.

[79] He, W.; Van Puyvelde, L.; Maes, L.; Bosselaers, J.; De Kimpe, N. Antitrichomonas in vitro activity of Cussonia holstii Engl. Nat. Prod. Res., 2003, 17(2), 127-133.

[80] Loyola, L.A.; Borquez, J.; Morales, G.; Araya, J.; Gonzalez, J.; Neira, I.; Sagua, H.; San-Martin, A. Diterpenoids from Azorella yareta and their trichomonicidal activities. Phytochemistry, 2001, 56(2), 177-180.

(C) Gehrig and Efferth; Licensee Bentham Open .

This is an open access article licensed under the terms of the Creative Commons Attribution Non-Commercial License (http://creativecommons.org/licenses/by-nc/3.0/) which permits unrestricted, non-commercial use, distribution and reproduction in any medium, provided the work is properly cited. 